\title{
Damage evolution in particulate composite materials
}

\author{
Karel Matouš * \\ Department of Mechanical Aerospace and Nuclear Engineering, Rensselaer Polytechnic Institute, 110 8th Street, Troy, NY 12180, USA
}

Received 19 April 2002; received in revised form 18 November 2002

\begin{abstract}
Damage evolution in heterogeneous solids is modeled using transformation field analysis and imperfect interface model. Stress changes caused by local debonding are simulated by residual stresses generated by equivalent transformation strains or eigenstrains. Decohesion and both overall and local stress and strain rates are derived from thermodynamics of irreversible processes, which provide an excellent framework for the development of constitutive equations. Both tangent and unloading secant stiffness tensors are found along any prescribed mechanical loading path. Numerical simulation of debonding evolution in glass/elastomer composites is compared with experimental data and provides good agreement between the model and experiments.
\end{abstract}

(c) 2002 Elsevier Science Ltd. All rights reserved.

Keywords: Particulate reinforced composites; Damage evolution; Debonding; Energy release rate; Thermodynamics

\section{Introduction}

Particulate composites represent a large group of materials used in a variety of applications, such as automobile tires and/or solid rocket propellant. The mechanical behavior of these materials depends upon properties of constituents and any microstructural changes that may occur in the body under loading. The main source of such changes in a material can be a nonlinear response of a matrix and/or damage. The damage in reinforced composites appears to be associated with microcracks initiating and growing within a matrix and along the matrix-particle interfaces. The mechanical behavior of these phenomena has been studied extensively over many years: Farris (1968) studied vacuole formation and growth; Mullins (1969) discovered the Mullins effect; Schapery (1999), Park and Schapery (1997), Ha and Schapery (1998) and Simo (1987) studied viscoelasticity with growing damage; Swanson and Christensen (1983) described strain softening; Christensen and Lo (1979) proposed models for stiffness reduction in coated particles and/or fibers, and Hashin (1991) provided analytical expressions of local and overall stress and strain fields as functions of the phase moduli and interfacial properties. Interface friction or sliding theories have been also investigated by Hutchinson and Jensen (1990). Another group of models published in recent years devoted to progressive interfacial decohesion of composite and other material systems has been proposed as well by Krajcinovic (1996) and Chaboche et al. (2001). However, the micromechanics-based models have not been

\footnotetext{
${ }^{*}$ Tel.: +1-518-276-6204; fax: +1-518-276-6025.

E-mail address: matouk@rpi.edu (K. Matouš).
} 
fully utilized yet in damage evolution, except in finite element methods of unit cell models described, for example, by Zhong and Knauss (1997).

The present work follows up the article published by Dvorak and Zhang (2001), who presented a procedure for analysis of progressive damage in composite materials, which incorporates the local stress averages provided by Dvorak's transformation field analysis (TFA) method (Dvorak and Benveniste, 1992; Dvorak, 1992) involving certain influence functions. Here such analysis is extended from a stress-based into a strain-based approach, enriched by thermodynamics, and both total and rate formulations are developed. At the beginning of Section 2, we summarize principal equations of TFA and present decomposition of a volume fraction of a reinforcement to bonded and debonded phases. Decohesion of the particles is modeled by an equivalent eigenstrain, which modifies local stress fields in the affected volume to values implied by Hashin's imperfect interface model (Hashin, 1991). Section 3 illustrates the procedures for damage evolution law based on thermodynamics of irreversible processes, which provide an excellent framework for the development of constitutive equations. Such a framework has been extensively used during the past decade, in many different contexts (Chaboche, 1997; Ju, 1989; Lubarda and Krajcinovic, 1995). Finally, an application is made to a rubber reinforced composite with several different reinforcement densities, and compared to experiments performed by Vratsanos and Farris (1993).

The symbolic notation adopts lower-case boldface Greek letters e.g. $\sigma$ for $(6 \times 1)$ vectors representing second-order tensors, and upper-case boldface italics letters e.g. $\boldsymbol{S}, \boldsymbol{E}$ for $(6 \times 6)$ matrices representing fourth-order tensors. The symbol marked by bar, $\bar{V}$, denotes quantity $V$ at the overall level, so that $\overline{\boldsymbol{L}}$ denotes the overall stiffness matrix.

\section{Partially debonded elastic composite systems}

Consider a composite medium, shown in Fig. 1, at a certain time of the loading path, consisting of three phases: matrix, bonded and debonded particles, with representative volume fractions $c_{\mathrm{m}}+c_{\mathrm{b}}+c_{\mathrm{d}}=1$, $c_{2}=c_{\mathrm{b}}+c_{\mathrm{d}}$ where subscripts, $\mathrm{m}, \mathrm{b}, \mathrm{d}$, and 2, represent matrix, bonded, debonded phases and total reinforcement, respectively. For simplicity the superscript, $t$, which refers to the value at the current time $t \in \mathbb{R}^{+}$, has been dropped. The value of the volume fraction of debonded phases and its rate $c_{\mathrm{d}}, \dot{c}_{\mathrm{d}}$ are given by the corresponding damage condition and evolution law as shown later in the article. Two limit cases can be observed; for $c_{\mathrm{d}}=0$, reinforcement is fully bonded, and for $c_{\mathrm{d}} \rightarrow c_{2}, c_{\mathrm{b}} \rightarrow 0$, the system converts into a porous medium with a cavity volume fraction $c_{2}$ and overall elastic properties dependent only on the elastic moduli of a matrix. The local stress strain relation in a representative volume element (RVE), which is subdivided into certain sub-volumes $r, s=\mathrm{m}, \mathrm{b}, \mathrm{d}$ and loaded by uniform overall strain $\overline{\boldsymbol{\varepsilon}}$, reads,

$$
\boldsymbol{\sigma}_{r}=\boldsymbol{L}_{r}\left(\boldsymbol{\varepsilon}_{r}-\boldsymbol{\mu}_{r}\right), \quad \boldsymbol{\varepsilon}_{r}=\boldsymbol{A}_{r} \overline{\boldsymbol{\varepsilon}}+\sum_{s=1}^{3} \boldsymbol{D}_{r s} \boldsymbol{\mu}_{s}
$$

where $\boldsymbol{L}_{r}$ represents phase elastic stiffness and $\boldsymbol{\mu}_{r}$ denotes eigenstrain, which represents stress free strain induced in the system by thermal and/or inelastic effects. The $\boldsymbol{A}_{r}, \boldsymbol{D}_{r s}$ denote certain mechanical and transformation tensors, respectively. Evaluation of the mechanical $\boldsymbol{A}_{r}$ and concentration $\boldsymbol{D}_{r s}$ tensors for the three phase composite medium can be found in the studies by Dvorak and Benveniste (1992) and Dvorak (1992),

$$
\begin{aligned}
& \boldsymbol{A}_{r}=\boldsymbol{A}_{r}^{\mathrm{I}}\left(\sum_{s=1}^{3} c_{s} \boldsymbol{A}_{s}^{\mathrm{I}}\right)^{-1}=\left(\boldsymbol{L}^{*}+\boldsymbol{L}_{r}\right)^{-1}\left(\boldsymbol{L}^{*}+\overline{\boldsymbol{L}}\right), \quad \boldsymbol{A}_{s}^{\mathrm{I}}=\left(\boldsymbol{L}^{*}+\boldsymbol{L}_{s}\right)^{-1}\left(\boldsymbol{L}^{*}+\boldsymbol{L}_{0}\right) \\
& \boldsymbol{D}_{r s}=\left(\boldsymbol{I}-\boldsymbol{A}_{r}\right)\left(\boldsymbol{L}_{r}-\overline{\boldsymbol{L}}\right)^{-1}\left(\delta_{r s} \boldsymbol{I}-c_{s} \boldsymbol{A}_{s}^{\mathrm{T}}\right) \boldsymbol{L}_{s}
\end{aligned}
$$




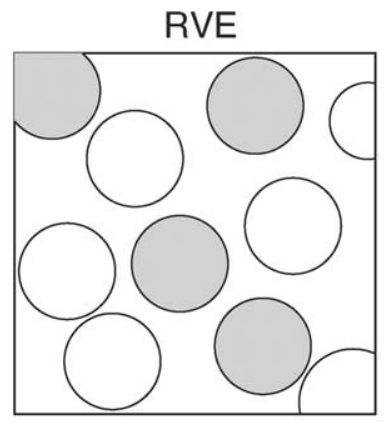

$\mathrm{t}$

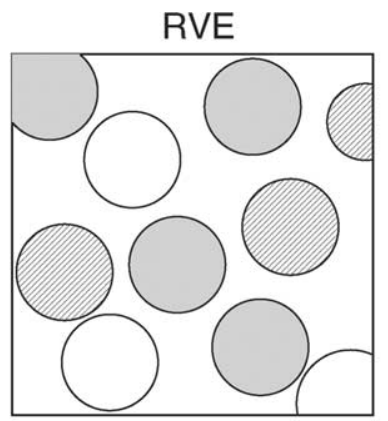

$t+\Delta t$
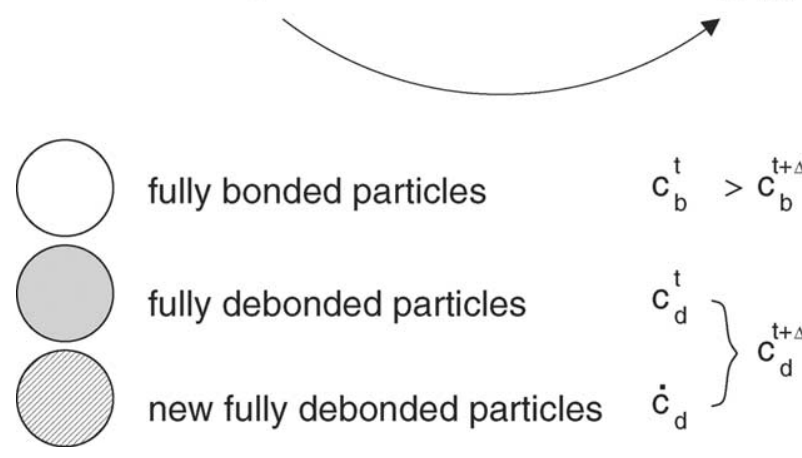

fully bonded particles

$$
c_{b}^{t}>c_{b}^{t+\Delta t}=c_{b}^{t} \Delta t \dot{c}_{d}
$$

fully debonded particles

new fully debonded particles

$$
\left.\begin{array}{l}
c_{d}^{t} \\
\dot{c}_{d}
\end{array}\right\} c_{d}^{t+\Delta t}=c_{d}^{t}+\Delta t \dot{c}_{d}
$$

Fig. 1. Damage evolution in a composite medium and decomposition of volume fraction of particles.

where in the present context, $\boldsymbol{L}_{\mathrm{b}}=\boldsymbol{L}_{\mathrm{d}}=\boldsymbol{L}_{2} ; \boldsymbol{A}_{\mathrm{b}}=\boldsymbol{A}_{\mathrm{d}}=\boldsymbol{A}_{2}, \overline{\boldsymbol{L}}$ denotes stiffness of the homogeneous material, and $\delta_{r s}$ represents the Kronecker symbol. The $\boldsymbol{A}_{2}$ tensor is evaluated in a perfectly bonded medium with Hill's constraint tensor $\boldsymbol{L}^{*}=\boldsymbol{L}_{0} \boldsymbol{S}^{-1}\left(\boldsymbol{I}-\boldsymbol{S}\right.$ ), where $\boldsymbol{S}$ is the Eshelby (Eshelby, 1957) tensor, and $\boldsymbol{L}_{0}$ denotes stiffness of a comparison medium; $\boldsymbol{A}_{r}^{\mathrm{I}}$ denotes partial mechanical concentration tensor usually used in the Mori-Tanaka method, where a matrix serves as a comparison medium $\boldsymbol{L}_{0}=\boldsymbol{L}_{\mathrm{m}}$, and $\boldsymbol{A}_{\mathrm{m}}^{\mathrm{I}}$ is equal to an identity matrix $\boldsymbol{I}$.

At the macroscale, the overall strain $\overline{\boldsymbol{\varepsilon}}$ and stress $\overline{\boldsymbol{\sigma}}$ are related by

$$
\begin{aligned}
& \overline{\boldsymbol{\varepsilon}}=c_{\mathrm{m}} \boldsymbol{\varepsilon}_{\mathrm{m}}+c_{\mathrm{b}} \boldsymbol{\varepsilon}_{\mathrm{b}}+c_{\mathrm{d}} \boldsymbol{\varepsilon}_{\mathrm{d}} \\
& \overline{\boldsymbol{\sigma}}=c_{\mathrm{m}} \boldsymbol{\sigma}_{\mathrm{m}}+c_{\mathrm{b}} \boldsymbol{\sigma}_{\mathrm{b}}+c_{\mathrm{d}} \boldsymbol{\sigma}_{\mathrm{d}}, \quad \overline{\boldsymbol{\sigma}}=\overline{\boldsymbol{L}}(\overline{\boldsymbol{\varepsilon}}-\overline{\boldsymbol{\mu}})
\end{aligned}
$$

and the overall eigenstrain $\bar{\mu}$ follows from Levin's formula,

$$
\overline{\boldsymbol{\mu}}=\sum_{r=1}^{3} c_{r} \boldsymbol{B}_{r}^{\mathrm{T}} \boldsymbol{\mu}_{r}, \quad \boldsymbol{B}_{r}=\left(\boldsymbol{M}^{*}+\boldsymbol{M}_{r}\right)^{-1}\left(\boldsymbol{M}^{*}+\overline{\boldsymbol{M}}\right)
$$

Note that compliance of a homogeneous system $\overline{\boldsymbol{M}}=\overline{\boldsymbol{L}}^{-1}$, compliance of a cavity $\boldsymbol{M}^{*}=\left(\boldsymbol{L}^{*}\right)^{-1}$, and concentration tensors $\boldsymbol{A}_{r}, \boldsymbol{B}_{r}$ must satisfy $\sum_{r=1}^{3} c_{r} \boldsymbol{A}_{r}=\sum_{r=1}^{3} c_{r} \boldsymbol{B}_{r}=\boldsymbol{I}$. Similar requirements for transformation influence tensors $\boldsymbol{D}_{r s}$ are described by Dvorak and Benveniste (1992).

\subsection{Overall stiffness and selection of comparison medium}

As pointed out by Dvorak and Srinivas (1999) a number of estimates of composite stiffness $\overline{\boldsymbol{L}}$ of any statistically homogeneous RVE consisting of $r=1,2, \ldots, N$ phases of the same shape and orientation, but possibly different sizes, can be written as, 


$$
\overline{\boldsymbol{L}}=\left[\sum_{r=1}^{N} c_{r}\left(\boldsymbol{L}^{*}+\boldsymbol{L}_{r}\right)^{-1}\right]^{-1}-\boldsymbol{L}^{*}
$$

Moreover, Walpole (1969a,b) proved that Eq. (5) satisfies Hashin and Shtrikman (1962) first-order variational bounds on the actual overall elastic properties, where bounds $\overline{\boldsymbol{L}}^{+}$and $\overline{\boldsymbol{L}}^{-}$on the actual $\overline{\boldsymbol{L}}$ are obtained by selecting the stiffness $\boldsymbol{L}_{0}$ of a comparison medium.

For particulate reinforced composite materials where progressive damage does not create entirely unsupported cavities and overall moduli depend still on $\boldsymbol{M}_{2}$, one can use for $0 \leqslant c_{\mathrm{d}} \leqslant c_{2}$ the comparison medium suggested by Dvorak and Srinivas (1999),

$$
\boldsymbol{L}_{0}=\alpha_{1} \boldsymbol{L}_{\mathrm{m}}+\alpha_{2} \boldsymbol{L}_{2}, \quad \alpha_{1}+\alpha_{2}=1
$$

However, in the medium that eventually becomes porous $c_{\mathrm{d}} \rightarrow c_{2}$, the overall stiffness $\overline{\boldsymbol{L}}$ might not depend on $\boldsymbol{M}_{2}$; therefore, as indicated by Dvorak and Zhang (2001) the definition of the comparison medium yields for $\phi>c_{2}$,

$$
\boldsymbol{L}_{0}=\boldsymbol{L}_{\mathrm{m}}\left[c_{\mathrm{m}}\left(1+\alpha c_{2}\right)\right], \quad 0<\alpha<1 / c_{\mathrm{m}}
$$

and for $0<\phi \leqslant c_{2}$,

$$
\boldsymbol{L}_{0}=\boldsymbol{L}_{\mathrm{m}}\left[\left(c_{\mathrm{m}} \phi\right)^{-1}\left(\phi-c_{2}\right)\right]
$$

where $\phi$ denotes the percolation threshold of a closely packed reinforcement and $c_{\mathrm{d}} \rightarrow \phi \leqslant c_{2}$. The upper limit on $\alpha$ in Eq. (7) gives $\boldsymbol{M}_{0}=\boldsymbol{M}_{\mathrm{m}}$, as in the Mori-Tanaka method, which provides a lower bound on the overall compliance of a porous media. The additional material constraint condition for percolation threshold $\phi$ can be obtained at the point of failure of the medium, as shown later in Section 4 . We recall that $\boldsymbol{L}_{0}$ enters the analysis only in evaluation of the constraint tensor $\boldsymbol{L}^{*}$, or the related Eshelby tensor $\boldsymbol{S}$. Selection of the comparison medium can be broadly divided into those that make $\boldsymbol{L}_{0}$ depend on $c_{\mathrm{d}}$ and possibly other parameters or those that keep $\boldsymbol{L}_{0}$ constant. The second choice with $\boldsymbol{L}_{0}$ constant, Eq. (6), through debonding process, was used in the present analysis.

\subsection{Total strain formulation}

Here we rearrange the equations originally proposed by Dvorak and Zhang (2001) from the total stress formulation into the total strain formulation. The equivalent eigenstrain $\boldsymbol{\mu}_{\mathrm{d}}$ induced in the sub-volume $V_{\mathrm{d}} \in V$ is used in evaluation of the local fields in a heterogeneous system under the progressive damage by interfacial debonding. The current value of the averaged stress in the debonded particles $\sigma_{\mathrm{d}}$ is obtained employing Hashin's (Hashin, 1991) imperfect interface model where,

$$
\boldsymbol{\sigma}_{\mathrm{d}}=\boldsymbol{W} \boldsymbol{\sigma}_{\mathrm{m}}
$$

and $\boldsymbol{W}$ denotes partial stress concentration factor with coefficients dependent on $\boldsymbol{M}_{\mathrm{m}}, \boldsymbol{M}_{2}$ and interface properties. If $\boldsymbol{W} \rightarrow \mathbf{0}$, no force transfer between matrix and debonded particles occurs and sudden debonding is modeled; moreover, partial debonding is not permitted by this model, which is in accordance with averaging scheme applied. The more detailed description of the imperfect interface model is summarized in Appendix A.

For the sake of simplicity we do not assume the thermal effect and inelastic deformation of constituents, $\boldsymbol{\mu}_{\mathrm{m}}=\boldsymbol{\mu}_{\mathrm{b}}=0$. Therefore, only one nonzero eigenstrain $\boldsymbol{\mu}_{\mathrm{d}}$ remains in the analysis after substituting Eq. (9) into Eq. (1) for stress in the debonded phases $\sigma_{\mathrm{d}}$,

$$
\boldsymbol{\mu}_{\mathrm{d}}=\boldsymbol{N}^{-1} \boldsymbol{R} \overline{\boldsymbol{\varepsilon}}, \quad \boldsymbol{N}=\boldsymbol{L}_{2}\left(\boldsymbol{D}_{\mathrm{dd}}-\boldsymbol{I}\right)-\boldsymbol{W} \boldsymbol{L}_{\mathrm{m}} \boldsymbol{D}_{\mathrm{md}}, \quad \boldsymbol{R}=\boldsymbol{W} \boldsymbol{L}_{\mathrm{m}} \boldsymbol{A}_{\mathrm{m}}-\boldsymbol{L}_{2} \boldsymbol{A}_{2}
$$


where it is important to recall that $\boldsymbol{A}_{\mathrm{b}}=\boldsymbol{A}_{\mathrm{d}}=\boldsymbol{A}_{2}$ continue to hold through the analysis, because they are evaluated on the initial configuration and damage being treated as the eigenstrain (the TFA choice). The final form of the local stresses in terms of the overall strain $\overline{\boldsymbol{\varepsilon}}$ and the current value of $c_{\mathrm{d}}$ are obtained employing Eq. (10) into Eq. (1) as

$$
\boldsymbol{\sigma}_{r}=\boldsymbol{L}_{r} \mathscr{A}_{r} \overline{\boldsymbol{\varepsilon}}, \quad r=\mathrm{m}, \mathrm{b}, \mathrm{d}
$$

where damage mechanical tensors yield

$$
\mathscr{A}_{r}=\boldsymbol{A}_{r}+\left(\boldsymbol{D}_{r \mathrm{~d}}-\delta_{r \mathrm{~d}} \boldsymbol{I}\right) \boldsymbol{N}^{-1} \boldsymbol{R} \text {. }
$$

The overall stress follows from Eq. (3) in the form,

$$
\overline{\boldsymbol{\sigma}}=\overline{\mathbb{L}} \overline{\boldsymbol{\varepsilon}}, \quad \overline{\mathbb{Z}}=\left[c_{\mathrm{m}} \boldsymbol{L}_{\mathrm{m}} \mathscr{A}_{\mathrm{m}}+c_{\mathrm{b}} \boldsymbol{L}_{2} \mathscr{A}_{\mathrm{b}}+c_{\mathrm{d}} \boldsymbol{L}_{2} \mathscr{A}_{\mathrm{d}}\right]
$$

where the secant stiffness tensor $\overline{\mathbb{L}}$ denotes a symmetric rank fourth-order unloading damage modulus. It is assumed that all interfacial cracks caused by debonding of particles from the matrix close upon unloading, and therefore no permanent deformation exists upon complete unloading. Fig. 2 shows overall elastic and secant modulus for 1D example, where one can observe that the gap between origin (point $O$ ) and point $A$ is actually the inelastic eigenstrain $\overline{\boldsymbol{\mu}}$ due to debonding during the loading process.

\subsection{Strain rate formulation}

The incremental strain approach is very important for successful implementation of the proposed model into a finite element environment. Moreover, the formulation of the tangent stiffness matrix is essential in successful implementation of the global iterative solver. Therefore, incremental constitutive equations and the tangent stiffness tensor are derived in the following section.

Taking the time derivative of constitutive equations (1) and keeping in mind that $\dot{\boldsymbol{\mu}}_{\mathrm{m}}=\dot{\boldsymbol{\mu}}_{\mathrm{b}}=\mathbf{0}$, we arrive at

$$
\begin{aligned}
& \dot{\boldsymbol{\sigma}}_{r}=\boldsymbol{L}_{r}\left(\dot{\boldsymbol{\varepsilon}}_{r}-\dot{\boldsymbol{\mu}}_{r}\right) \\
& \dot{\boldsymbol{\varepsilon}}_{r}=\boldsymbol{A}_{r} \dot{\overline{\boldsymbol{\varepsilon}}}+\dot{\boldsymbol{D}}_{r \mathrm{~d}} \boldsymbol{\mu}_{\mathrm{d}}+\boldsymbol{D}_{r \mathrm{~d}} \dot{\boldsymbol{\mu}}_{\mathrm{d}} \\
& \dot{\boldsymbol{\sigma}}_{r}=\boldsymbol{L}_{r} \boldsymbol{A}_{r} \dot{\overline{\boldsymbol{\varepsilon}}}+\boldsymbol{L}_{r} \dot{\boldsymbol{D}}_{r \mathrm{~d}} \boldsymbol{\mu}_{\mathrm{d}}+\boldsymbol{L}_{r}\left(\boldsymbol{D}_{r \mathrm{~d}}-\delta_{r \mathrm{~d}} \boldsymbol{I}\right) \dot{\boldsymbol{\mu}}_{\mathrm{d}}, \quad r=\mathrm{m}, \mathrm{b}, \mathrm{d}
\end{aligned}
$$

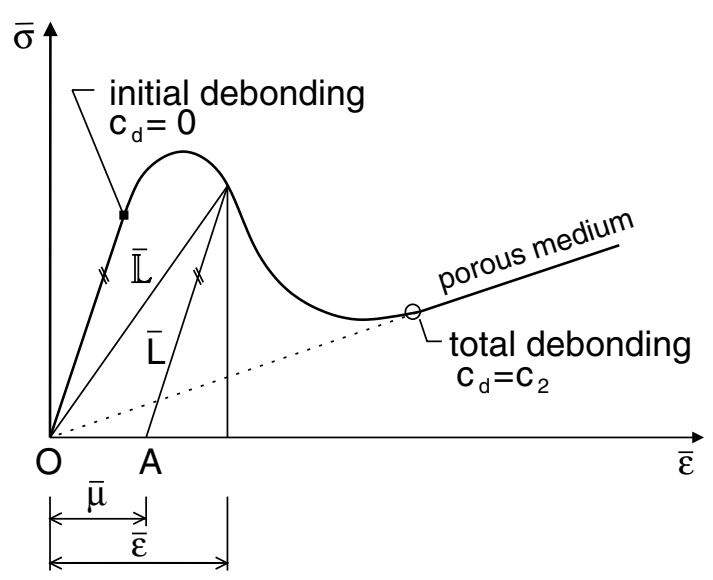

Fig. 2. Stress-strain curve for debonding model. 
where concentration tensors, Eq. (2) $)_{2}$, are functions of elastic moduli, mechanical tensors and volume fractions $c_{r}$. However, only volume fractions $c_{\mathrm{b}}, c_{\mathrm{d}}$ of fully bonded and debonded particles vary through time. Therefore, differentiation of $\boldsymbol{D}_{r \mathrm{~d}}\left(c_{\mathrm{d}}(t)\right)$ with respect to time takes the form

$$
\dot{\boldsymbol{D}}_{r \mathrm{~d}}=\frac{\partial \boldsymbol{D}_{r \mathrm{~d}}}{\partial c_{\mathrm{d}}} \dot{c}_{\mathrm{d}}=-\boldsymbol{Q}_{r} \boldsymbol{A}_{2}^{\mathrm{T}} \boldsymbol{L}_{2} \dot{c}_{\mathrm{d}}
$$

and $\boldsymbol{Q}_{r}=\left(\boldsymbol{I}-\boldsymbol{A}_{r}\right)\left(\boldsymbol{L}_{r}-\overline{\boldsymbol{L}}\right)^{-1}$. Similarly, taking the time derivative of the debonding eigenstrain $\boldsymbol{\mu}_{\mathrm{d}}$, Eq. (10), and using results from (15) we obtain

$$
\dot{\boldsymbol{\mu}}_{\mathrm{d}}=\boldsymbol{N}^{-1} \boldsymbol{R} \dot{\overline{\boldsymbol{\varepsilon}}}+\boldsymbol{N}^{-1}\left(\boldsymbol{L}_{2} \boldsymbol{Q}_{2}-\boldsymbol{W} \boldsymbol{L}_{\mathrm{m}} \boldsymbol{Q}_{\mathrm{m}}\right) \boldsymbol{A}_{2}^{\mathrm{T}} \boldsymbol{L}_{2} \boldsymbol{N}^{-1} \boldsymbol{R} \overline{\boldsymbol{\varepsilon}} \dot{c}_{\mathrm{d}}
$$

The incremental form of the local strain fields is then given by

$$
\begin{aligned}
& \dot{\boldsymbol{\varepsilon}}_{\mathrm{m}}=\mathscr{A}_{\mathrm{m}} \dot{\overline{\boldsymbol{\varepsilon}}}+\mathscr{B}_{\mathrm{m}} \overline{\bar{\varepsilon}} \dot{c}_{\mathrm{d}} \\
& \dot{\boldsymbol{\varepsilon}}_{\mathrm{b}}=\mathscr{A}_{\mathrm{b}} \dot{\overline{\boldsymbol{\varepsilon}}}+\mathscr{B}_{\mathrm{b}} \dot{\bar{\varepsilon}}_{\mathrm{c}} \\
& \dot{\boldsymbol{\varepsilon}}_{\mathrm{d}}=\widetilde{\mathscr{A}}_{\mathrm{d}} \dot{\overline{\boldsymbol{\varepsilon}}}+\widetilde{\mathscr{B}}_{\mathrm{d}} \overline{\mathrm{c}}_{\mathrm{c}} \dot{\mathrm{c}}_{\mathrm{d}}
\end{aligned}
$$

and local incremental constitutive equations $(14)_{1}$ read,

$$
\dot{\boldsymbol{\sigma}}_{r}=\boldsymbol{L}_{r} \mathscr{A}_{r} \dot{\overline{\boldsymbol{\varepsilon}}}+\boldsymbol{L}_{r} \mathscr{B}_{r} \overline{\bar{\varepsilon}} \dot{c}_{\mathrm{d}}, \quad r=\mathrm{m}, \mathrm{b}, \mathrm{d}
$$

where

$$
\begin{aligned}
& \mathscr{B}_{r}=\left[\left(\boldsymbol{D}_{r \mathrm{~d}}-\delta_{r \mathrm{~d}} \boldsymbol{I}\right) \boldsymbol{N}^{-1}\left(\boldsymbol{L}_{2} \boldsymbol{Q}_{2}-\boldsymbol{W} \boldsymbol{L}_{\mathrm{m}} \boldsymbol{Q}_{\mathrm{m}}\right)-\boldsymbol{Q}_{r}\right] \boldsymbol{A}_{2}^{\mathrm{T}} \boldsymbol{L}_{2} \boldsymbol{N}^{-1} \boldsymbol{R} \\
& \widetilde{\mathscr{A}}_{\mathrm{d}}=\boldsymbol{A}_{\mathrm{d}}+\boldsymbol{D}_{\mathrm{dd}} \boldsymbol{N}^{-1} \boldsymbol{R} \\
& \widetilde{\mathscr{B}}_{\mathrm{d}}=\left[\boldsymbol{D}_{\mathrm{dd}} \boldsymbol{N}^{-1}\left(\boldsymbol{L}_{2} \boldsymbol{Q}_{2}-\boldsymbol{W} \boldsymbol{L}_{\mathrm{m}} \boldsymbol{Q}_{\mathrm{m}}\right)-\boldsymbol{Q}_{\mathrm{d}}\right] \boldsymbol{A}_{2}^{\mathrm{T}} \boldsymbol{L}_{2} \boldsymbol{N}^{-1} \boldsymbol{R}
\end{aligned}
$$

\section{Damage evolution}

The proposed approach employs irreversible thermodynamics and the internal state variables theory to describe a damage evolution process in particulate reinforced composites. Similar formulations have been applied to a variety of continuum damage mechanics problems, (Ju, 1989; Simo and Ju, 1987a,b; Lemaitre, 1985; Lubarda and Krajcinovic, 1995); however, most of these formulations deal only with homogeneous or homogenized materials.

To introduce damage behavior, let us consider the free energy potential assuming small deformations as,

$$
\Psi\left(\overline{\boldsymbol{\varepsilon}}, c_{\mathrm{d}}\right)=\frac{1}{2} \overline{\boldsymbol{\varepsilon}}^{\mathrm{T}} \overline{\mathbb{\complement}} \overline{\boldsymbol{\varepsilon}}
$$

Confining our attention to the purely mechanical theory, the Clausius-Duhem inequality reads,

$$
-\dot{\Psi}+\overline{\boldsymbol{\sigma}}^{\mathrm{T}} \dot{\overline{\boldsymbol{\varepsilon}}} \geqslant 0
$$

so that dissipative inequality arrives after taking the time derivative of (20) as,

$$
\mathfrak{D} \equiv-\frac{1}{2} \overline{\boldsymbol{\varepsilon}}^{\mathrm{T}} \dot{\overline{\mathbb{L}}} \overline{\boldsymbol{\varepsilon}}=-\frac{1}{2} \overline{\boldsymbol{\varepsilon}}^{\mathrm{T}} \mathscr{J} \overline{\boldsymbol{\varepsilon}}_{\mathrm{d}}=Y \dot{\boldsymbol{c}}_{\mathrm{d}} \geqslant 0
$$

where

$$
\mathscr{J}=c_{\mathrm{m}} \boldsymbol{L}_{\mathrm{m}} \mathscr{B}_{\mathrm{m}}+c_{\mathrm{b}} \boldsymbol{L}_{2} \mathscr{B}_{\mathrm{b}}+c_{\mathrm{d}} \boldsymbol{L}_{2} \mathscr{B}_{\mathrm{d}}+\boldsymbol{L}_{2}\left[\left(\boldsymbol{D}_{\mathrm{dd}}-\boldsymbol{I}\right)-\boldsymbol{D}_{b d}\right] \boldsymbol{N}^{-1} \boldsymbol{R}
$$

Differentiation of the overall secant stiffness tensor $\overline{\mathbb{L}}$ in Eq. (20) with respect to $c_{\mathrm{d}}$ requires some mathematical algebra; one has to differentiate complicated damage mechanical tensors $\mathscr{A}_{r}$, Eq. (12), which 
depend on concentration tensors $\boldsymbol{D}_{r \mathrm{~d}}\left(c_{\mathrm{d}}(t)\right)$, respectively. The proposed thermodynamic force (damage energy release rate) conjugated to the damage volume fraction $c_{\mathrm{d}}$ yields,

$$
Y=-\frac{1}{2} \overline{\boldsymbol{\varepsilon}}^{\mathrm{T}} \mathscr{J} \overline{\boldsymbol{\varepsilon}}
$$

Significant similarity can be observed with the thermodynamic force $Y=-(1 / 2) \overline{\boldsymbol{\varepsilon}}^{\mathrm{T}} \overline{\boldsymbol{L}} \overline{\boldsymbol{\varepsilon}}$ proposed by Ju (1989) for homogeneous material and the isotropic damage model characterized by damage potential in the form $\Psi(\overline{\boldsymbol{\varepsilon}}, \omega)=(1-\omega)(1 / 2) \overline{\boldsymbol{\varepsilon}}^{\mathrm{T}} \overline{\boldsymbol{L}} \overline{\boldsymbol{\varepsilon}}$, where $\omega$ denotes the isotropic damage variable. However, the main difference comes from the operator $\mathscr{J}$, which is derived from implemented micromechanical analysis.

To define the onset or continuation of debonding, one can adopt the approach based on a damage surface customarily introduced (Ju, 1989; Bittnar and Šjnoha, 1996) in an analogy to the yield surface of the theory of plasticity. The state of damage in the material is characterized by the damage criterion given by,

$$
g\left(Y, \chi^{t}\right)=G(Y)-\chi^{t} \leqslant 0, \quad t \in \mathbb{R}^{+}
$$

where $\chi^{t}$ denotes the softening parameter usually set as $\chi^{t=0}=0$. The function $G(Y)$ that characterizes the damage process in the material can be given by the Weibull distribution,

$$
G(Y)=c_{2}-c_{2} \exp \left[-\left(\frac{Y-Y_{\text {in }}}{p_{1} Y_{\text {in }}}\right)^{p_{2}}\right]
$$

where $Y_{\text {in }}$ denotes the initial threshold (energy barrier), and $p_{1}$ and $p_{2}$ are scale and shape parameters.

The damage potential $\Psi\left(\overline{\boldsymbol{\varepsilon}}, c_{\mathrm{d}}\right)$ depends on $c_{\mathrm{d}}$ in a complicated way, as shown by the expression (23), and the thermodynamic force $Y$ cannot be expressed as a simple function of $\Psi$. Nevertheless, the initial energy barrier $Y_{\text {in }} \equiv Y\left(c_{\mathrm{d}}=0, \overline{\boldsymbol{\varepsilon}}\right)$ can be estimated from experimentally measured overall strain $\overline{\boldsymbol{\varepsilon}}$ and free energy per unit volume stored in systems with different reinforcement densities before the first debonding occurs and is assumed to be a material parameter. As will be shown, free energy per unit volume stored in the system does not differ too much for composites with low and high concentration of particles. Such observation leads to the conclusion to use only two initial thermodynamic forces for all reinforcement spectrum.

Indeed, damage initiation can take place only if a nucleation stress exceeds a critical value, (which is approximately equal to Young's modulus of a rubber as a result of a local geometrical instability as shown in Gent and Lindley (1958)). Although the nucleation criterion is satisfied a priori due to the experimentally measured initial energy release rate $Y_{\text {in }}$, an additional condition to (25) is defined for the surface averaged interfacial normal stress,

$$
\sigma^{\text {in }}>0
$$

Eq. (27) shows that if hydrostatic pressure is applied $\sigma^{\text {in }}<0$ while the material is deformed, the pressure will not initiate a void formation in the present approach. Thus proposed model describes damage evolution for any loading path where surface averaged interfacial normal stress $\sigma^{\text {in }}$ is tensile and damaged material converts into a porous medium. A similar damage function to Eq. (25) can be used for damage evolution in compressive regime, $\sigma^{\text {in }}<0$, if such and/or similar damage mechanism exists. In such a case, the system converts into a debonded particulate filled material and debonded particles can still carry part of compressive forces. This behavior can be simulated by calibration of interface properties used in partial stress concentration factor $\boldsymbol{W}$ (see Eq. (9) and Appendix A).

The damage process is derived in terms of the following irreversible, dissipative equation of evolution,

$$
\dot{c}_{\mathrm{d}}=\dot{\kappa} \frac{\partial g}{\partial Y}=\dot{\kappa} H, \quad H=\frac{\partial G(Y)}{\partial Y}
$$


where $\kappa$ is a damage consistency parameter, which defines damage loading-unloading conditions according to Kuhn-Tucker relations,

$$
\dot{\kappa} \geqslant 0, \quad g\left(Y, \chi^{t}\right) \leqslant 0, \quad \dot{\kappa} g\left(Y, \chi^{t}\right) \equiv 0
$$

In addition, we define that $\dot{\chi}^{t}=\dot{\kappa} H$, and the parameter $\kappa$ is determined from the consistency condition $\dot{g}=0$, from which follows,

$$
\dot{\kappa}=\dot{Y}, \quad \dot{Y}=-\overline{\boldsymbol{\varepsilon}}^{\mathrm{T}} \mathscr{J} \dot{\overline{\boldsymbol{\varepsilon}}}-\frac{1}{2} \overline{\boldsymbol{\varepsilon}}^{\mathrm{T}} \mathscr{K} \overline{\boldsymbol{\varepsilon}} \dot{c}_{\mathrm{d}}
$$

where

$$
\mathscr{K}=2 \mathscr{J} \boldsymbol{R}^{-1}\left(\boldsymbol{L}_{2} \boldsymbol{Q}_{2}-\boldsymbol{W} \boldsymbol{L}_{\mathrm{m}} \boldsymbol{Q}_{\mathrm{m}}\right) \boldsymbol{A}_{2}^{\mathrm{T}} \boldsymbol{L}_{2} \boldsymbol{N}^{-1} \boldsymbol{R}
$$

After introducing Eqs. (31) and (30) into (28), the increment of damage volume fraction in matrix notation leads to,

$$
\dot{c}_{\mathrm{d}}=-\frac{H}{1+\frac{H}{2} \overline{\boldsymbol{\varepsilon}}^{\mathrm{T}} \mathscr{K} \overline{\bar{\varepsilon}}} \overline{\overline{\boldsymbol{\varepsilon}}}^{\mathrm{T}} \mathscr{J} \dot{\overline{\boldsymbol{\varepsilon}}}=\mathscr{P}^{\mathrm{T}} \dot{\overline{\boldsymbol{\varepsilon}}}
$$

Please note that the vector $\mathscr{P}^{\mathrm{T}}$ of dimension $(1 \times 6)$ represents a second-order tensor so that notation $\mathscr{P}^{T} \dot{\overline{\boldsymbol{\varepsilon}}}$ reads $\mathscr{P}: \dot{\overline{\boldsymbol{\varepsilon}}}$. Eq. (32) can now be used to relate the local strain $\dot{\boldsymbol{\varepsilon}}_{r}$ and stress $\dot{\boldsymbol{\sigma}}_{r}$ rates to the overall strain rate $\dot{\bar{\varepsilon}}$ as,

$$
\begin{aligned}
& \dot{\boldsymbol{\varepsilon}}_{\mathrm{m}}=\left[\mathscr{A}_{\mathrm{m}}+\mathscr{B}_{\mathrm{m}} \overline{\boldsymbol{\varepsilon}} \mathscr{P}^{\mathrm{T}}\right] \dot{\overline{\boldsymbol{\varepsilon}}} \\
& \dot{\boldsymbol{\varepsilon}}_{\mathrm{b}}=\left[\mathscr{A}_{\mathrm{b}}+\mathscr{B}_{\mathrm{b}} \overline{\overline{\boldsymbol{\varepsilon}}} \mathscr{P}^{\mathrm{T}}\right] \dot{\overline{\boldsymbol{\varepsilon}}} \\
& \dot{\boldsymbol{\varepsilon}}_{\mathrm{d}}=\left[\widetilde{\mathscr{A}}_{\mathrm{d}}+\widetilde{\mathscr{B}}_{\mathrm{d}} \overline{\overline{\boldsymbol{\varepsilon}}} \mathscr{P}^{\mathrm{T}}\right] \dot{\overline{\boldsymbol{\varepsilon}}}
\end{aligned}
$$

where matrix notation $\overline{\boldsymbol{\varepsilon}} \mathscr{P}^{\mathrm{T}}(6 \times 1)(1 \times 6)=(6 \times 6)$ corresponds to its tensor equivalent $\overline{\boldsymbol{\varepsilon}} \otimes \mathscr{P}$, and

$$
\dot{\boldsymbol{\sigma}}_{r}=\left[\boldsymbol{L}_{r} \mathscr{A}_{r}+\boldsymbol{L}_{r} \mathscr{B}_{r} \overline{\boldsymbol{\varepsilon}} \mathscr{P}^{\mathrm{T}}\right] \dot{\overline{\boldsymbol{\varepsilon}}}, \quad \dot{\boldsymbol{\sigma}}_{r}=\mathscr{L}_{r} \dot{\overline{\boldsymbol{\varepsilon}}}, \quad r=\mathrm{m}, \mathrm{b}, \mathrm{d}
$$

Finally, when employing the equation of equilibrium written in terms of phase stresses and phase volume fractions,

$$
\dot{\overline{\boldsymbol{\sigma}}}=c_{\mathrm{m}} \dot{\boldsymbol{\sigma}}_{\mathrm{m}}+c_{\mathrm{b}} \dot{\boldsymbol{\sigma}}_{\mathrm{b}}+c_{\mathrm{d}} \dot{\boldsymbol{\sigma}}_{\mathrm{d}}+\left(\boldsymbol{\sigma}_{\mathrm{d}}-\boldsymbol{\sigma}_{\mathrm{b}}\right) \dot{\boldsymbol{c}}_{\mathrm{d}}, \quad \dot{\overline{\boldsymbol{\sigma}}}=\overline{\mathscr{L}} \dot{\overline{\boldsymbol{\varepsilon}}}
$$

we arrive at the desired form of the overall tangent stiffness matrix,

$$
\overline{\mathscr{L}}=\left[c_{\mathrm{m}} \mathscr{L}_{\mathrm{m}}+c_{\mathrm{b}} \mathscr{L}_{\mathrm{b}}+c_{\mathrm{d}} \mathscr{L}_{\mathrm{d}}+\boldsymbol{L}_{2}\left(\mathscr{A}_{\mathrm{d}}-\mathscr{A}_{\mathrm{b}}\right) \overline{\boldsymbol{\varepsilon}}^{\mathrm{P}}\right]
$$

This form of the material tangent stiffness modulus can be used in the Newton-Raphson method to provide the equilibrium of the system for a given load increment. The resulting modulus is a symmetric rank fourthorder tensor; the symmetry depends crucially on the form of the thermodynamic force (Simo and $\mathrm{Ju}$, 1987 a,b). In fact, it is very difficult to prove that $\overline{\mathscr{L}}$ is diagonally symmetric, and alternative definition of Eq. (24) would result in the non-symmetric tangent operator.

It follows that an accurate response prediction requires an incremental analysis. Specifically, the overall as well as local stresses corresponding to the $j+1$ th increment yield,

$$
\begin{aligned}
& \overline{\boldsymbol{\sigma}}^{j+1}=\overline{\boldsymbol{\sigma}}^{j}+\overline{\mathscr{L}} \Delta \overline{\boldsymbol{\varepsilon}} \\
& \boldsymbol{\sigma}_{r}^{j+1}=\boldsymbol{\sigma}_{r}^{j}+\mathscr{L}_{r} \Delta \overline{\boldsymbol{\varepsilon}}
\end{aligned}
$$

and the internal state variable (damage volume fraction) is provided by implicit Euler backward integration,

$$
c_{\mathrm{d}}^{j+1}=c_{\mathrm{d}}^{j}+\mathscr{P}^{\mathrm{T}} \Delta \overline{\boldsymbol{\varepsilon}}
$$


Finding the increment $\Delta c_{\mathrm{d}}=\mathscr{P}^{\mathrm{T}} \Delta \overline{\boldsymbol{\varepsilon}}$ of the debonded volume fraction $c_{\mathrm{d}}^{j+1}$ for a given loading increment leads to an iterative procedure,

$$
{ }^{k+1}\left(\Delta c_{\mathrm{d}}\right)=-\frac{{ }^{k} H}{1+\frac{{ }^{k} H}{2}{ }^{k} \overline{\boldsymbol{\varepsilon}}^{\mathrm{T} k} \mathscr{K}^{k} \overline{\boldsymbol{\varepsilon}}}{ }^{k} \overline{\boldsymbol{\varepsilon}}^{\mathrm{T} k} \mathscr{J} \Delta \overline{\boldsymbol{\varepsilon}}={ }^{k} \mathscr{P}^{\mathrm{T}} \Delta \overline{\boldsymbol{\varepsilon}}
$$

and usually three to five iterations $k$ are sufficient to reach the convergence criterion,

$$
\frac{\left\|^{k+1}\left(\Delta c_{\mathrm{d}}\right)-{ }^{k}\left(\Delta c_{\mathrm{d}}\right)\right\|}{\left\|{ }^{k}\left(\Delta c_{\mathrm{d}}\right)\right\|} \leqslant \text { tol }
$$

\section{Examples}

As an illustration and verification of the proposed model, we analyzed simple tension experiments by Vratsanos and Farris (1993) on polyurethane reinforced by spherical S-glass particles $60 \mu \mathrm{m}$ in radius with the standard deviation of the particle size of 0.042 , which corresponds to a very narrow size distribution. Concentrations of $c_{2}=0.12,0.24,0.48$ and 0.58 were used in the analysis. The average elastic moduli of the unfilled matrix and the elastic moduli of the reinforcement are listed in Table 1. All constituents were regarded as homogeneous isotropic elastic solids. For a good agreement with the measured overall elastic properties of all concentrations $c_{2}$, we selected the comparison medium (6) with $\alpha_{1}$ and $\alpha_{2}$ shown in the fifth and sixth column of Table 1, which also presents the elastic moduli of the homogenized composite system obtained from Eq. (5). The very low magnitude of spring constants $\xi=\eta=0.1 \mathrm{MPa} / \mathrm{m}$ was selected to simulate a sudden complete debonding of particles.

The particular choice of this material was motivated by the availability of experimental data for different reinforcement densities. Although experiments show the presence of large deformations (at least for low densities of reinforcement), which are not included by the proposed theory, the comparison of measurements and predictions is in good agreement, and the present model provides novel findings into the damage evolution in rubber reinforced composite materials. In general, the experimental data shows more curvature in stress-strain curves. This curvature is most likely caused by the behavior of the matrix. The unfilled rubber is assumed to act as a linear elastic solid in the present model, and all deviations from linearity are assumed to be due to debonding. Therefore, extension of the model using the nonlinear viscoelasticity or other constitutive laws for rubber will improve the agreement.

The material scale $p_{1}=6.4$ and shape $p_{2}=0.9$ parameters used in the damage function, Eq. (26), were obtained by fitting the measured stress strain curve for the $24 \%$ reinforcement density. The initial energy release rate $Y_{\text {in }}$ was estimated using the overall strain $\overline{\boldsymbol{\varepsilon}}$ and the free energy per volume stored in the system before first debonding occurred, Eq. (24), where $c_{\mathrm{d}}=0$. Two values of the initial thermodynamic force were used for the low and high reinforcement densities, as $Y_{\text {in }}(12-24 \%)=0.085 \mathrm{MJ} / \mathrm{m}^{3}$ and $Y_{\text {in }}(48-58 \%)=0.018 \mathrm{MJ} / \mathrm{m}^{3}$,

Table 1

Constituent and overall elastic properties of S-glass/polyurethane composites

\begin{tabular}{llllll}
\hline Material & $E(\mathrm{MPa})$ & $G(\mathrm{MPa})$ & $v$ & $\alpha_{1}$ & $\alpha_{2}$ \\
\hline S-glass & $86.8 \times 10^{3}$ & $35.0 \times 10^{3}$ & 0.24 & - & - \\
Polyurethane & 0.49 & 0.164 & 0.49 & - & - \\
Composite, $c_{2}(\%)$ & & & 0.483 & 0.99998281 & 0.00001719 \\
12 & 0.981 & 0.331 & 0.468 & 0.99996907 & 0.00003093 \\
24 & 2.174 & 0.740 & 0.462 & 0.99998246 & 0.00001754 \\
48 & 3.817 & 1.305 & 0.405 & 0.99993001 & 0.00006999 \\
58 & 13.830 & 4.921 & & & \\
\hline
\end{tabular}




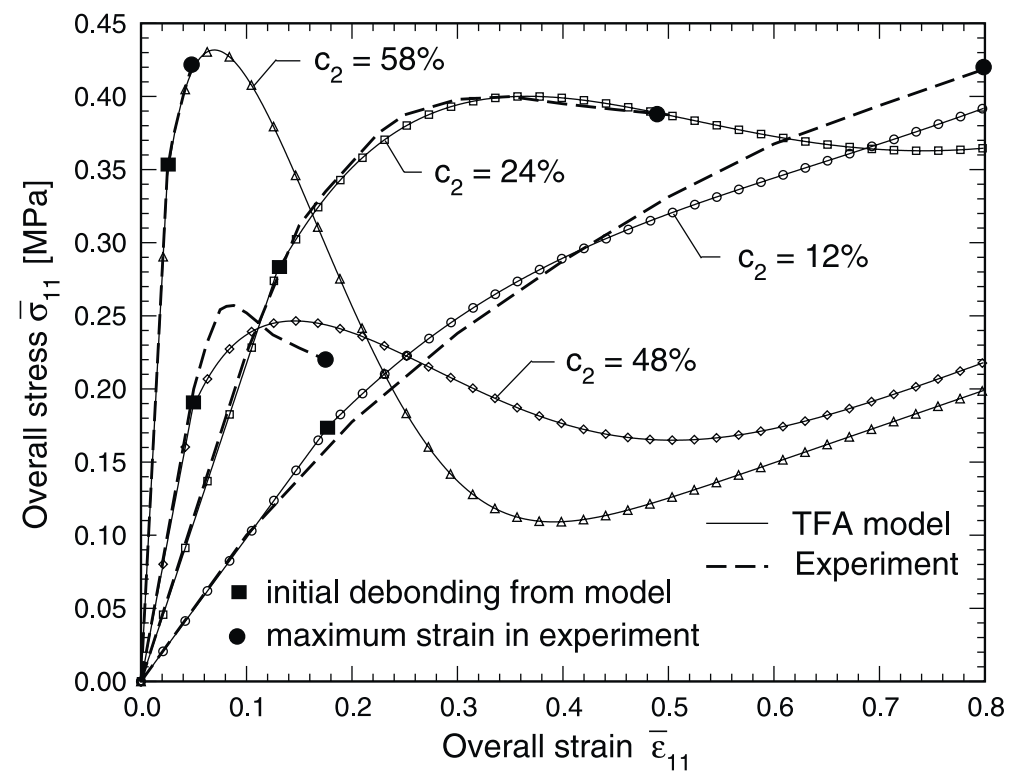

Fig. 3. Comparison of the predicted and measured overall response of S-glass/polyurethane composite at four reinforcement densities and the standard deviation of the particle size distribution 0.042 .

respectively. All the above material parameters were fixed in the numerical analysis. In what follows, the presented results are obtained employing simple integration algorithm given by Eqs. (37)-(40).

Fig. 3 displays comparisons of the experimental and predicted tensile stress-strain curves for the four reinforcement volume fractions. Only one result for the particle density of $c_{2}=48 \%$ does not match the experimental data perfectly. Possible causes of this anomalous behavior were discussed in Vratsanos and Farris (1993) and can be given for example by poor curing of this particular specimen. Other possibilities were discussed in Dvorak and Zhang (2001).

Table 2 shows the corresponding computed overall strains, stresses, and energies per unit volume stored in the system when the first debonding takes place. One can see that energies stored in the system do not differ rapidly for the two low and the two high densities, respectively. Therefore, only two initial thermodynamic forces were selected as described above. However, a substantial difference in energy between the low and high concentrations is observed. It is evident that the energy stored in the system necessary for debonding decreases as the material density increases, and thus the system becomes more brittle.

Fig. 4 shows the propagation of damage as a function of the overall strain and volume fraction, suggesting a much more progressive debonding, or apparently brittle response of the highly reinforced and thus much stiffer materials. To show the capability of the present model, the complete loading path from a fully bonded to a porous medium is depicted in both Figs. 3 and 4. However, rupture of specimens occurred

Table 2

Strain, stress and energy potential at first debonding

\begin{tabular}{lllll}
\hline & \multicolumn{1}{c}{$c_{2}(\%)$} & & \\
\cline { 2 - 5 } & 12 & 24 & 48 & 58 \\
\hline $\bar{\varepsilon}_{11}(\mathrm{MPa})$ & 0.178 & 0.130 & 0.049 & 0.025 \\
$\bar{\sigma}_{11}\left(\mathrm{MJ} / \mathrm{m}^{3}\right)$ & 0.175 & 0.283 & 0.187 & 0.346 \\
$\Psi(0.0184$ & 0.0045 & 0.0043 \\
\hline
\end{tabular}




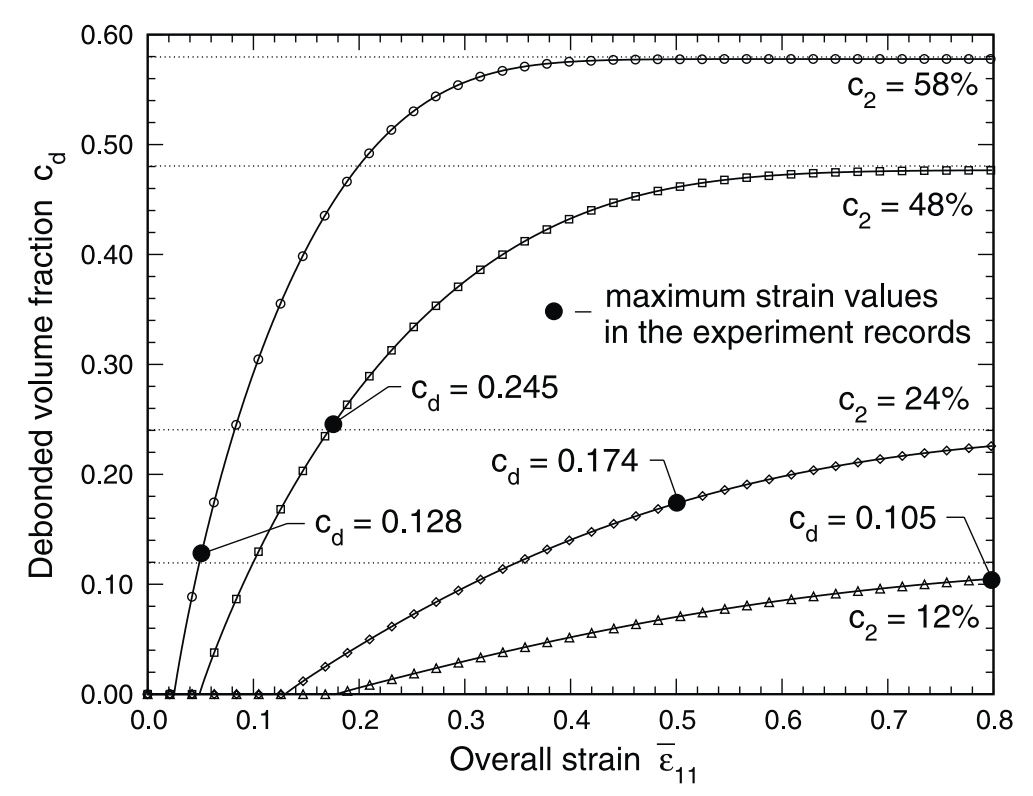

Fig. 4. Evolution of debonding as a function of the overall tensile strain $\overline{\boldsymbol{\varepsilon}}_{11}$.

much earlier before decohesion of all particles. A possible explanation for such behavior is the strain localization caused by the clustering of particles especially for high densities, in which a material becomes more brittle and particles have no time to fully debond before the material ruptures.

At the point of failure of the material, a linear dependency between the percentage of debonded particles and the total volume fraction of reinforcement in the composite was observed (Fig. 5). This important

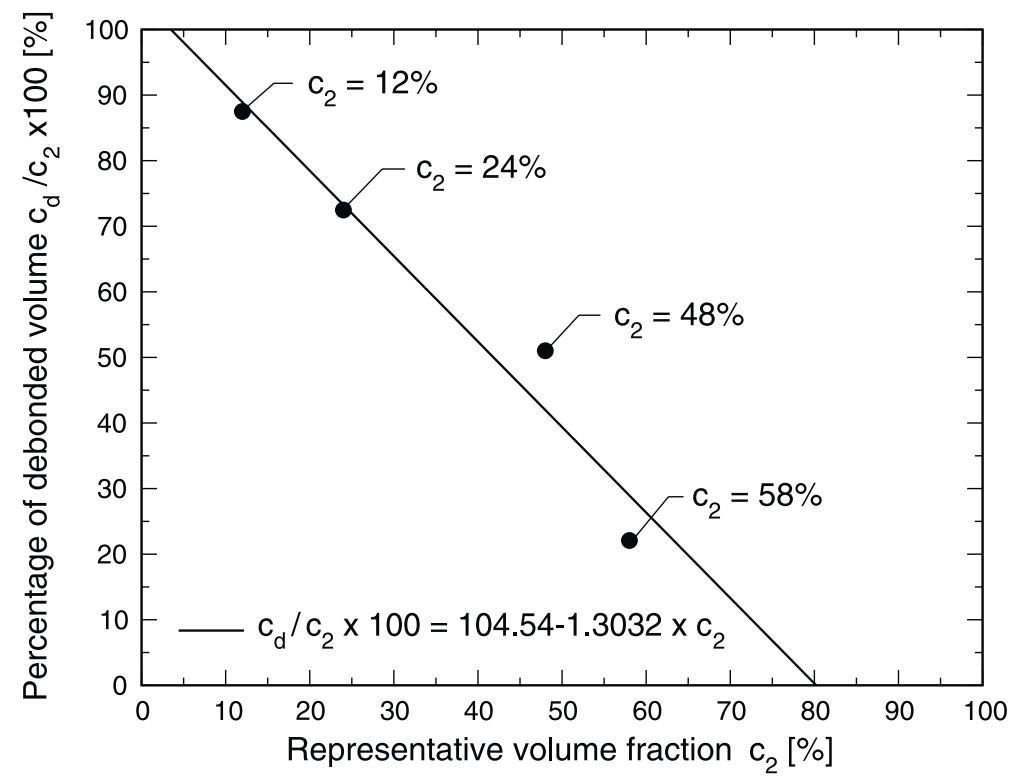

Fig. 5. Percentage of debonded particles in the system at the rupture of specimen. 

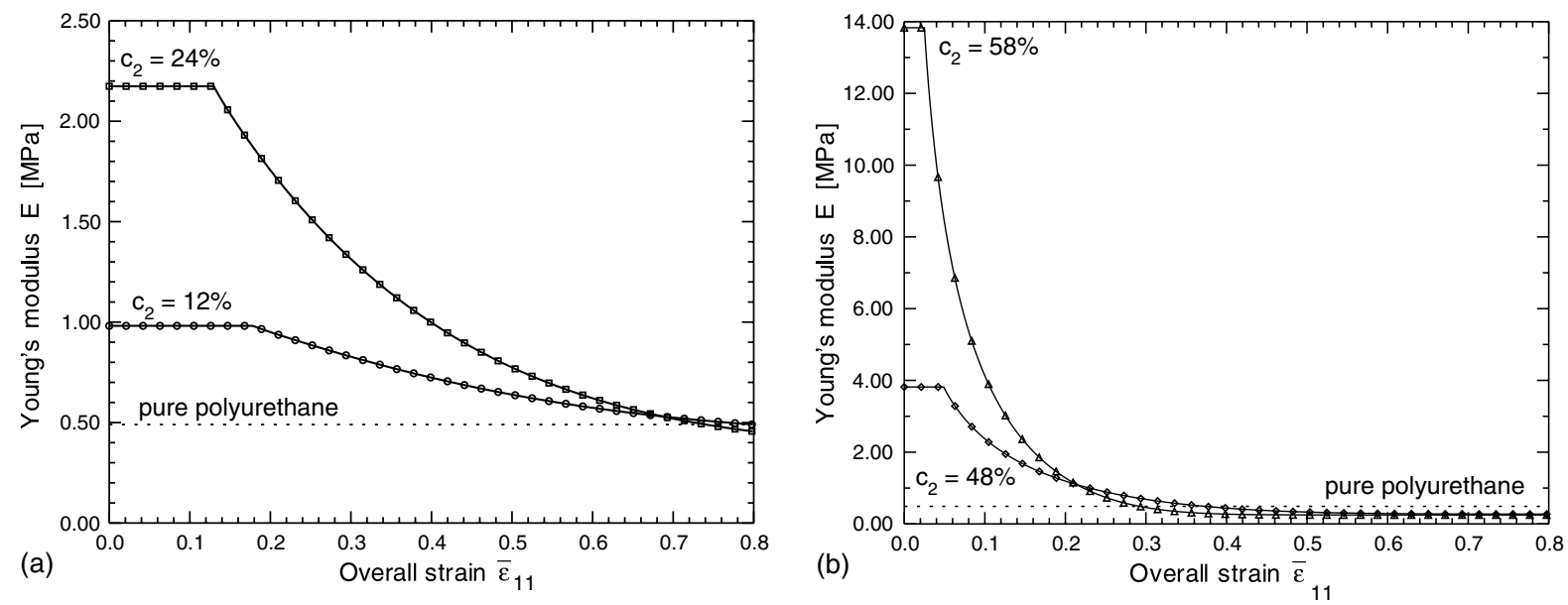

Fig. 6. Degradation of the elastic modulus as a function of the overall tensile strain $\overline{\boldsymbol{\varepsilon}}_{11}$.

finding shows that for low densities almost all particles debond before rupture and thus a material becomes porous and the fracture is very similar to ductile. On the other hand, when a high reinforcement density is present in a material, the system is more brittle, and thus only a small percentage of particles debond before a sudden rupture appears. A similar discovery was presented by Vratsanos and Farris (1993). One can use this finding to formulate the additional material constraint condition for the percolation threshold of a closely packed reinforcement $\phi$, used in Eq. (8), and limit damage evolution as $c_{\mathrm{d}} \rightarrow \phi<c_{2}$, so that limit stress and strain at the failure can be determined for $c_{\mathrm{d}}=\phi$.

Fig. 6 shows degradation of the elastic modulus as a function of the overall tensile strain. As expected, slow degradation is observed for $12 \%$ reinforcement, where stiffness of the porous material is very close to the pure polyurethane. The rapid change in Young's modulus is obtained for 58\% volume fraction, so that the material converts to the porous medium with material properties lower that those for the unfilled matrix.

\section{Conclusion}

The proposed mathematical model, based on Dvorak's TFA together with thermodynamics of irreversible processes and the internal state variables theory, which induces sufficient constraints against a set of possibilities which is too large, is shown to describe successfully the damage evolution in particulate reinforced elastomers. Stress changes caused by local debonding are simulated by residual stresses generated by equivalent transformation strains or eigenstrains, which are derived from Hashin's imperfect interface spring-layer model. The energy release rate is derived from the free energy function, and both the total and incremental strain-based formulations, including loading tangent and unloading secant stiffness tensors, are found for any loading path.

The current numerical approach is limited to small deformations; however, good agreement between the model and experiments for the uniaxial tension test performed by Vratsanos and Farris (1993) was obtained for several densities of reinforcement. The material completely ruptures before decohesion of all particles, especially for high reinforcement densities, and thus much stiffer and brittle system. However, for low densities almost all particles debond before rupture, so that the material becomes porous and the fracture is 
very similar to ductile. Based on such observations, the material constraint condition for the percolation threshold of a closely packed reinforcement is proposed and limits the total decohesion.

Further study is required to extend the model to cover the nonlinear deformation of matrix. Moreover, implementation of the theory into a finite element code is necessary for the solution of complex geometry and/or boundary and loading conditions.

\section{Acknowledgement}

I wish to thank Professor G.J. Dvorak for helpful discussions.

\section{Appendix A}

Here we describe the imperfect interface spring-layer model proposed by Hashin (1991). The geometry and loading conditions are shown in Fig. 7. Both the particle, d, and matrix, $\mathrm{m}$, are isotropic elastic solids, with elastic properties $E_{\mathrm{m}}, G_{\mathrm{m}}, v_{\mathrm{m}}$ and $E_{2}, G_{2}, v_{2}$. The interface traction-displacement relations are prescribed as,

$$
T_{\rho}=\eta\left[u_{\rho}\right], \quad T_{\theta}=\xi\left[u_{\theta}\right], \quad T_{\varphi}=\xi\left[u_{\varphi}\right]
$$

where $\eta$ and $\xi$ are the normal and tangential spring constants of the interface, respectively. The displacement jumps across the interface are represented by $\left[u_{\rho, \theta, \varphi}\right]$. The average stress in the inclusion can be found by integration of local stress distribution $\hat{\boldsymbol{\sigma}}_{\mathrm{d}}$ as,

$$
\boldsymbol{\sigma}_{\mathrm{d}}=\frac{1}{V_{\mathrm{d}}} \int_{V} \hat{\boldsymbol{\sigma}}_{\mathrm{d}} \mathrm{d} V=\frac{3}{4 \pi} \int_{0}^{\pi} \mathrm{d} \varphi \int_{0}^{\pi} \sin \theta \mathrm{d} \theta \int_{0}^{1} \hat{\boldsymbol{\sigma}}_{\mathrm{d}} \beta^{2} \mathrm{~d} \beta
$$

where $\beta=\rho / a$ is the normalized radial distance. The result can be, according to Hashin, reduced to the matrix form required in Eq. (9),
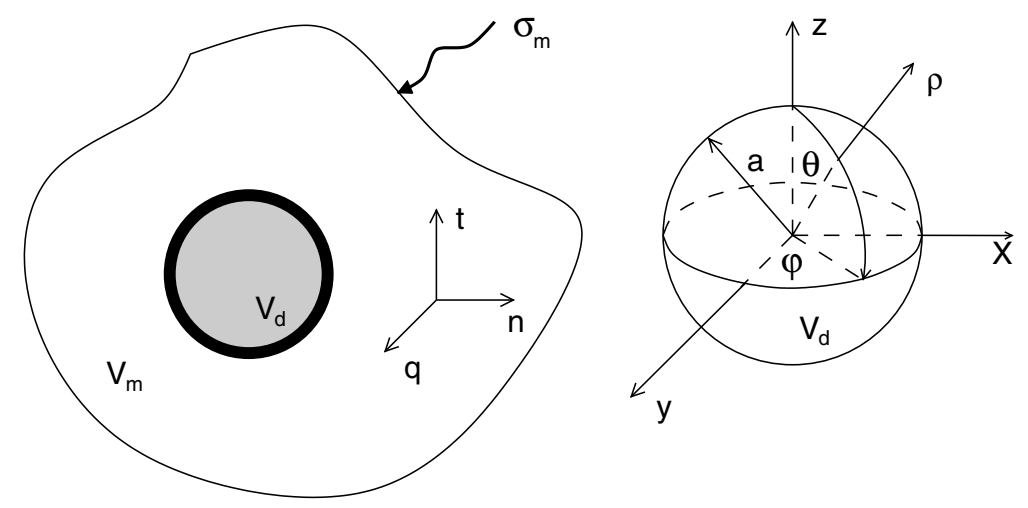

Fig. 7. A spherical inclusion with imperfect interface. 


$$
\boldsymbol{\sigma}_{\mathrm{d}}=\boldsymbol{W} \boldsymbol{\sigma}_{\mathrm{m}}, \quad \mathbf{W}=\left[\begin{array}{rrrrrr}
\gamma+\frac{2 \vartheta}{3} & \gamma-\frac{\vartheta}{3} & \gamma-\frac{\vartheta}{3} & 0 & 0 & 0 \\
& \gamma-\frac{\vartheta}{3} & \gamma-\frac{\vartheta}{3} & 0 & 0 & 0 \\
& & \gamma-\frac{\vartheta}{3} & 0 & 0 & 0 \\
& & & \vartheta & 0 & 0 \\
& & & & \vartheta & 0 \\
\text { sym } & & & & \vartheta
\end{array}\right]
$$

where $\vartheta=G_{2}\left(21 A_{2}+5 B_{2}\right) / 5 G_{\mathrm{m}}, \gamma=K_{2}\left(3 K_{\mathrm{m}}+4 G_{\mathrm{m}}\right) /\left[3 K_{\mathrm{m}}\left(3 K_{2}+4 G_{\mathrm{m}}(1+3 \imath)\right)\right], K_{r}$ is a bulk modulus, and $\imath=K_{2} /(\eta a)$ denotes the interface parameter. Constants $A_{2}, B_{2}$ can be obtained from the following system of equations,

$$
\left[\begin{array}{cccc}
2\left(5-v_{\mathrm{m}}\right) & -6 & -3 v_{2} h & h \\
-2\left(1+v_{\mathrm{m}}\right) & 4 & \left(7+2 v_{2}\right) h & h \\
-2\left(5-4 v_{\mathrm{m}}\right) & 3 & 12 v_{2}(1-e) h & 2(1+2 e) \\
-2\left(1-2 v_{\mathrm{m}}\right) & -1 & \left(7-4 v_{2}\right)+\left(7+2 v_{2}\right) 2 f & 1+2 f
\end{array}\right]\left\{\begin{array}{l}
C_{1} \\
D_{1} \\
A_{2} \\
B_{2}
\end{array}\right\}=\left\{\begin{array}{l}
1 \\
1 \\
2 \\
1
\end{array}\right\}
$$

where $f=G_{2} / a \xi, h=G_{\mathrm{m}} / G_{2}$ and $e=G_{2} / a \eta$.

\section{References}

Bittnar, Z., Šejnoha, J., 1996. Numerical Methods in Structural Engineering. ASCE Press.

Chaboche, J.L., 1997. Thermodynamic formulation of constitutive equations and application to the viscoplasticity and viscoelasticity of metals and polymers. Int. J. Solids Struct. 34 (18), 2239-2254.

Chaboche, J.L., Kruch, S., Maire, J.F., Poittier, T., 2001. Towards a micromechanics based inelastic and damage modeling of composites. Int. J. Plasticity 17, 411-439.

Christensen, R.N., Lo, K.H., 1979. Solutions for effective properties of composite materials. J. Mech. Phys. Solids 27, 315-330.

Dvorak, G.J., Benveniste, Y., 1992. On transformation strains and uniform fields in multiphase elastic media. Proc. R. Soc. London A 437, 291-310.

Dvorak, G.J., 1992. Transformation field analysis of inelastic composite materials. Proc. R. Soc. London A $437,311-327$.

Dvorak, G.J., Srinivas, M.V., 1999. New estimates of overall properties of heterogeneous solids. J. Mech. Phys. Solids 47, 899-920.

Dvorak, G.J., Zhang, J., 2001. Transformation field analysis of damage evolution in composite materials. J. Mech. Phys. Solids 49, 2517-2541.

Eshelby, J.D., 1957. The determination of the elastic field of an ellipsoidal inclusion and related problems. Proc. R. Soc. London A 241, 376-396.

Farris, R.J., 1968. The character of the stress-strain function for highly filled elastomers. Trans. Soc. Rheol. 12, 303-314.

Gent, A.N., Lindley, P.B., 1958. Internal rupture of bonded rubber cylinders in tension. Proc. R. Soc. A 249, $195-205$.

Ha, K., Schapery, R.A., 1998. A three-dimensional viscoelastic constitutive model for particulate composites with growing damage and its experimental validation. Int. J. Solids Struct. 26-27, 3497-3517.

Hashin, Z., Shtrikman, S., 1962. On some variational principles in anisotropic and nonhomogeneous elasticity. J. Mech. Phys. Solids $10,335-342$.

Hashin, Z., 1991. The spherical inclusion with imperfect interface. ASME J. Appl. Mech. 58, 444-449.

Hutchinson, J.W., Jensen, H.M., 1990. Models of fiber debonding and pullout in brittle composites with friction. Mech. Mater. 9, 335442.

Ju, J.W., 1989. On energy-based coupled elastoplastic damage theories: constitutive modeling and computational aspects. Int. J. Solids Struct. 25 (7), 803-833.

Krajcinovic, D., 1996. Damage Mechanics. Elsevier Science, Amsterdam, p. 750.

Lemaitre, J., 1985. Coupled elasto-plastic and damage constitutive equations. Comp. Meth. Appl. Mech. Eng. 51, 31-49.

Lubarda, V.A., Krajcinovic, D., 1995. Some fundamentals issues in rate theory of damage-elastoplasticity. Int. J. Plasticity 11 (7), $763-797$.

Mullins, L., 1969. Softening of rubber by deformation. Rubber Chem. Tech. 42, 339-361. 
Park, S.W., Schapery, 1997. A viscoelastic constitutive model for particulate composites with growing damage. Int. J. Solids Struct. 34 (8), 931-947.

Schapery, R.A., 1999. Nonlinear viscoelastic and viscoplastic constitutive equations with growing damage. Int. J. Fract. 97, 33-66. Simo, J.C., Ju, J.W., 1987a. Strain- and stress-based continuum damage models-I. Formulation. Int. J. Solids Struct. 23 (7), 821-840. Simo, J.C., Ju, J.W., 1987b. Strain- and stress-based continuum damage models-II. Computational aspects. Int. J. Solids Struct. 23 (7), 841-869.

Simo, J.C., 1987. On a fully three-dimensional finite-strain viscoelastic damage model: formulation and computational aspects. Comp. Meth. Appl. Mech. Eng. 60, 153-173.

Swanson, S.R., Christensen, L.W., 1983. A constitutive formulation for high-elongation propellants. J. Spacecraft Rockets 20, 559566.

Vratsanos, V.A., Farris, R.J., 1993. A predictive model for the mechanical behavior of particulate composites. Part I: model derivation. Polym. Eng. Sci. 33 (22), 1458-1465.

Walpole, L.J., 1969a. On bounds for the overall elastic moduli of inhomogeneous system I. J. Mech. Phys. Solids 14, 151-162.

Walpole, L.J., 1969b. On bounds for the overall elastic moduli of inhomogeneous system II. J. Mech. Phys. Solids 14, $289-301$.

Zhong, X.A., Knauss, W.G., 1997. Analysis of interfacial failure in particle-filled elastomer. ASME J. Eng. Mater. Tech. 119, 198-204. 